\title{
A Case of Hyperosmolar Hyperglycemic State Caused by Ischemic Pancreatitis
}

\author{
Erika Leung, MD*; Keith W. Murdock, MD; Richard W. Lustig, DO;Heath G. Wilt, DO \\ University of Missouri-Kansas City; Department of Internal Medicine; St. Luke's Hospital; \\ Kansas City, Missouri, USA
}

\begin{abstract}
Background: The hyperosmolar hyperglycemic state (HHS) is a dangerous complication that can arise with diabetes mellitus. Emergent treatment is necessary to reinstate hemodynamic stability, as mortality rates for HHS are exceptionally high and can have multiple complications.

Case Report: A 42-year-old female presented unresponsive to the emergency department. Upon arrival, the patient's examination was consistent with hypovolemia and tachycardia. Review of systems included a one-week history of nausea and vomiting. The patient had stopped all of her medications one year prior, only restarting metformin one week prior to admission. Her presenting systolic blood pressure was $119 \mathrm{mmHg}$ before she developed respiratory failure secondary to a poor mental state. The presenting blood sugar level was $1263 \mathrm{mg} / \mathrm{dL}$, and ketones were not detected in her urine. The patient recovered from her HHS and was discharged in a stable condition.

Conclusion: This case study postulates that the patient's excessive nausea and vomiting led to pancreatitis secondary to ischemia, which induced a HHS. This is important because hyperglycemia superimposed on any ischemic insult can increase the risk of multiorgan failure, as with our patient. Thus, recognition and management of resulting complications needs to be emergent.
\end{abstract}

Keywords: diabetes mellitus; sepsis, ischemia; hyperglycemia.

\section{Introduction}

The hyperosmolar hyperglycemic state (HHS) is one of the worst complications that can arise with diabetes mellitus; it is characterized by extreme hyperglycemia, increased serum osmolality, and prolonged dehydration in the absence of ketoacidosis [1]. In this situation, emergent treatment is necessary to reinstate hemodynamic stability. The mortality rate for HHS is exceptionally high and can have multiple side effects. According to Chen et al. [2], a hospital-based analysis illustrated a case fatality rate for HHS of $18.83 \%$ over the course of 28 days.

This case illustrates the various complications that can arise from HHS, emphasizing the necessity for careful

*Corresponding author: Erika Leung, MD. University of Missouri-Kansas City; Department of Internal Medicine; St. Luke's Hospital; Kansas City, Missouri, USA.

E-mail: Erika.leung@mail.mcgill.ca monitoring during the inpatient stay. The initiating event in HHS is glucosuric diuresis, which leads to free water loss and intravascular hyperosmolarity, this can exacerbate or cause acute kidney injury due to dehydration $[3,4]$. Traditionally HHS and diabetic ketoacidosis have been described as two separate states, but one-third of the patients have symptoms from both conditions [5]. While our case illustrates HHS arising from ischemic pancreatitis, other precipitating factors can include infections, medications, non-compliance, undiagnosed diabetes, substance abuse and other coexisting diseases [3]. Infections such as pneumonia, gastroenteritis, and urinary tract infections can also be a common cause.

\section{Case Description}

A 42-year-old unresponsive Caucasian female with a BMI of 35.0 was transferred to our facility with altered mental status and elevated blood glucose levels. She had experienced a one-week history of flu-like symptoms as well as nausea and hematemesis. Moreover, she had not taken insulin for over one 
year and had stopped her metformin for a month. She restarted her metformin a few days prior to this episode, which may have induced her nausea and vomiting. Upon arrival to our facility, her blood glucose level was reported to be $1263 \mathrm{mg} / \mathrm{dL}$. Her past medical history was relevant for type II diabetes mellitus, chronic back pain, hypothyroidism, hypertriglyceridemia, bipolar disorder, asthma and tobacco use.

Her initial laboratory values obtained from our hospital were as follows: her white blood cell count was $19.24 \times 10^{3}$ cells $/ \mathrm{mm}^{3}$, hemoglobin was $15.2 \mathrm{~g} / \mathrm{dL}$, sodium was 160 $\mathrm{mEq} / \mathrm{L}$, potassium was $3.9 \mathrm{mEq} / \mathrm{L}$, chloride was $122 \mathrm{mEq} / \mathrm{L}$, bicarbonate was $15 \mathrm{mEq} / \mathrm{L}$, BUN - $60 \mathrm{mg} / \mathrm{dL}$, creatinine - 2.4 $\mathrm{mg} / \mathrm{dL}$, glucose was rechecked and was $723 \mathrm{mg} / \mathrm{dL}$, creatine kinase of $1039 \mathrm{U} / \mathrm{L}$, amylase of $980 \mathrm{U} / \mathrm{L}$, lipase of $9992 \mathrm{U} / \mathrm{L}$, and triglycerides were $646 \mathrm{mg} / \mathrm{dL}$. She had an anion gap of 23. Her arterial blood gas resulted in a $\mathrm{pH}$ of 7.30 , a $\mathrm{pCO} 2$ of 36, a pO2 of 76 and bicarbonate of 17. Due to her elevated lipase and a CT scan suggestive of pancreatitis, the cause of her HHS was likely due to volume depletion, which led to ischemic pancreatitis.

The initial management was aggressive fluid resuscitation along with boluses of normal saline and an insulin drip. She was then admitted to the intensive care unit because her blood pressure had dropped to $91 / 54 \mathrm{mmHg}$ and her respiratory rate escalated to 42 , requiring intubation and vasopressors. A week into her hospitalization, her blood culture was positive for staphylococcus, with symptoms of systemic inflammatory response syndrome. The patient recovered from her HHS and her other various coexisting illnesses and was discharged in a stable condition.

\section{Discussion}

This was an unusual case of HHS, caused by ischemic pancreatitis, secondary to her metformin-induced nausea and vomiting, although her concomitant positive blood culture could have aggravated her state, as infections are predisposing factors for HHS [6]. However, because she suffered from subsequent distributive shock and was hypotensive requiring pressors, this could have also exacerbated her condition.

It is well documented in the literature that metformin can cause a rare and potentially fatal side effect, lactic acidosis. However, a more recent Cochrane review of 347 articles (2010) showed that there was no evidence based on prospective comparative trials or observational cohort studies that metformin increased the risk for lactic acidosis compared to any other antihyperglycemic medications [7]. In the 70,490 patient-years of the pooled metformin group, there were no cases of fatal or non-fatal lactic acidosis found [7]. Moreover, according to Chan et al. [8], the low prevalence of metforminassociated lactic acidosis was comparable to the prevalence of sulfonylurea-induced hypoglycemia; metformin had many benefits for management of type II diabetes mellitus, assuming that recommended guidelines for metformin use were adhered to [8]. Lalau et al. [9] also performed a literature search on the link between metformin and lactic acidosis in metformin therapy; and there was no true metformin-associated lactic acidosis and no mortalities due to metformin alone were found. Thus, it was ruled out that this patient's acidosis and HHS were caused by or induced by the use of metformin.

Sepsis and shock are serious manifestations of HHS, for if the patient develops these, prompt and proper treatment is needed as they have a high mortality rate, especially if the patient has various risk factors such as bacteremia, age greater than 65 years, immunosuppression, community acquired pneumonia, and genetic factors [10]. Fluid therapy needs to be administered as soon as possible, and because this patient was also suffering from ischemic pancreatitis, these two situations likely exacerbated one another; therefore, the need for intravascular repletion with fluids was even more vital [10]. Our patient experienced distributive shock as a consequence of her ischemic state, further leading to her multiorgan failure.

This case was an important review, as HHS is a lifethreatening condition which needs to be treated immediately. HHS can be easily overlooked; thus, this discussion becomes important when assessing how to properly and quickly treat these patients. There are many factors that can cause or provoke this condition. Moreover, it is uncommon for ischemic pancreatitis to be a source of HHS, which adds to the complexity of this case study. Some of the salient features of this case report include the questionable and irregular onset of HHS, its cause by ischemic pancreatitis, and other contributing factors, namely, sepsis concurrent with her recent restarting of metformin. Usually, the intake of metformin would lead to a side effect of lactic acidosis, which would induce diabetic ketoacidosis; however, in this case it may have led to HHS. Finally, this patient developed sepsis throughout her hospitalization while suffering from HHS. Both sepsis and HHS are conditions which can lead to immediate mortality, and when they occur simultaneously, critical and attentive care is vital for the patient. Mortality rates for both HHS and DKA are exceptionally high, and any additional condition can exacerbate the already severe situation. It is important to always monitor the HHS patients, as infections of any kind can increase mortality through these mechanisms. HHS is a state which has a high mortality if not monitored properly, and can worsen if any comorbidities exist, such as sepsis or ischemic pancreatitis.

\section{Competing interests}

The authors declare that they have no competing interests.

\section{References}

1. Davis SN, Umpierrez GE. Diabetic ketoacidosis in type 2 diabetes mellitus--pathophysiology and clinical presentation. Nat Clin Pract Endocrinol Metab 2007; 3(11): 730-1.

2. Chen HF, Wang CY, Lee HY, See TT, Chen MH, Jiang JY, et al. Short-term case fatality rate and associated factors among inpatients with diabetic ketoacidosis and hyperglycemic hyperosmolar state: a hospital-based analysis over a 15-year period. Intern Med 2010; 49(8): 729-37.

3. Stoner GD. Hyperosmolar hyperglycemic state. Am Fam Physician 2005; 71(9): 1723-30.

4. Matz R. Management of the hyperosmolar hyperglycemic syndrome. Am Fam Physician 1999; 60(5): 1468-76.

5. MacIsaac RJ, Lee LY, McNeil KJ, Tsalamandris C, 
Jerums G. Influence of age on the presentation and outcome of acidotic and hyperosmolar diabetic emergencies. Intern Med J 2002; 32(8):379-85.

6. Kitabchi AE, Umpierrez GE, Murphy MB, Barrett EJ, Kreisberg RA, Malone JI, et al. Management of hyperglycemic crises in patients with diabetes. Diabetes Care 2001;24(1): 131-53.

7. Salpeter S, Greyber E, Pasternak G, Salpeter E. Risk of fatal and nonfatal lactic acidosis with metformin use in type 2 diabetes mellitus." Cochrane Database Syst Rev 2003; (2):CD002967.

8. Chan NN, Brain HP, Feher MD. Metformin-associated lactic acidosis: a rare or very rare clinical entity? Diabet Med 1999; 16(4):273-81.

9. Lalau JD. Lactic acidosis induced by metformin: incidence, management and prevention. Drug Saf 2010; 33(9): 727-40. 